\title{
Teletherapy for Adolescent Psychiatric Outpatients: The Soaring Flight of so far Idle Technologies during the COVID-19 Pandemic
}

\author{
Mercedes M. Huscsava1*, Paul L. Plener ${ }^{1,2} \&$ Oswald D. Kothgassner ${ }^{1}$ \\ ${ }^{1}$ Department of Child and Adolescent Psychiatry, Medical University of Vienna, Währinger Gürtel 18-20, 1090 Vienna, Austria \\ ${ }^{2}$ Department of Child- and Adolescent Psychiatry and Psychotherapy, Medical University of Ulm, Steinhövelstraße 5, 89075 Ulm, Germany
}

\begin{abstract}
Background: As a consequence of the COVID-19 pandemic, teletherapeutic offers for patients skyrocketed. In a moment of great need, offers were emerging seemingly faster than the background technology and training required to facilitate them could be provided.

Objective: This Spotlight-Communication provides an overview of results and ideas on teletherapeutic offers for adolescent psychiatric outpatients and areas for further investigation.

Methods: It reports the insights gained from conducting follow-up interviews with 30 adolescents in weekly outpatient treatment Data were analyzed qualitatively using standard software ATLAS.ti 8 . Therapists' views were collected via informal discussions and are, although not systematically analyzed, integrated where applicable.

Results: On average, patients rated teletherapy as compared to face-to-face contacts as rather good to mediocre (3,2 on a 5-point-Likert scale). Main positive aspects were accessibility and continuity of treatment, as well as an "anchor"-function. Main negative aspects were technical issues, a lack of privacy and therapy being more superficial.

Conclusions: Although the results are not generalizable, they show that patients overall benefit from teletherapy. The main positives and pitfalls of teletherapy are described, as well as ideas for problem-solving and refinement, which are of utmost importance in light of potential further waves of the COVID-19 pandemic.
\end{abstract}

Keywords: child and adolescent psychiatry, adolescents, teletherapy, COVID-19, outpatients

\section{Article History}

Received 7 July 2020

Revised 17 August 2020

Accepted 17 August 2020

DOI 10.24989/dp.v1i2.1867

\section{Introduction}

The COVID-19 pandemic hit countries worldwide and confronted systems, as well as individuals, with enormous challenges in almost every aspect of life. Decision making processes had to be adapted at an extremely rapid pace, with almost no prior warning or preparation. In this rapidly changing situation, communication and the dissemination of information proved to be crucial, yet extremely challenging. In the midst of all this turmoil, already marginalized groups, like psychiatric patients, were deemed to be at an increased risk of disproportional harm. (Cui et al., 2020; Druss, 2020; Fegert et al., 2020; Kavoor, 2020; Yao et al., 2020) Thus, much effort, creativity and innovation went into establishing structures to comply with regulations and control spreading of the virus on the one hand, but facilitate continued treatment of those in need on the other. Basically overnight, teletherapy became the number one treatment option and is still on the rise. (Liu et al., 2020; Torous et al., 2020; Torous \& Wykes, 2020; Wind et al., 2020; Zho et al., 2020) Although the genesis of teletherapy was not the COVID-19 pandemic, its widespread application is definitely owed to it. Our clinic, the Department of Child and Adolescent Psychiatry, Medical University of Vienna, a tertiary care center and university hospital, switched its outpatient services overnight to therapy via video-call, keeping face-to-face contact as an option if absolutely unavoidable (acute presentations). Staff eager to serve their patients went into the "experiment" teletherapy without prior training or experience, being confronted not only with insecurity in relation to the technical aspects of this treatment, but also concerning the dynamic of the situation and resulting needs of the patients. Teletherapy as discussed herein refers to planned, individual videocalls only, other applications (e.g. emergency services, online-trainings) have not been examined.

\section{Aim and method}

The aim of this Spotlight-Communication is to concisely discuss patients', as well as psychiatrists' and psychologists', experiences 
with teletherapy, including positives, pitfalls and opportunities for future development. Given the novelty of the situation and the velocity of change, there is not a large volume of prior research to build upon in investigating diverse aspects of the current crisis. This article was thus conceptualized as brief communication discussing a small number of results from an ongoing investigation (Huscsava et al., 2020), integrating further ideas in a spotlight format. We followed up 30 mostly female patients $(86,7 \%)$, ranging from 12 to 18 years of age, who were in established weekly outpatient treatment before the onset of the COVID-19 pandemic, in the process of transition into teletherapy. Teletherapy, in the sense of planned, individual videocalls, was conducted using Instahelp (brand of Insta Communications $\mathrm{GmbH} / \mathrm{Up}$ to Eleven Digital Solutions $\mathrm{GmbH}$ ), an existing platform conforming to Austrian data safeguarding measures. In addition to videocalls, the platform also provides the function of an end-to-end encrypted individual chat. Ethical approval for this investigation was obtained from the institutional review board of the Medical University of Vienna (\#1383/2020) and patients and guardians gave written consent for participation in the study. Data were collected via interviews - videocall or phone call, according to the respective patient's preference - with outpatients in well-established, continuous weekly treatment before the COVID-19-associated lockdown. Interviews, conducted by two 5-year residents in Child and Adolescent Psychiatry, were semi-structured including open questions and questions to be rated on 3-, 4-, or 5-point Likert-scales. A description of the interview content, as well as sample characteristics and detailed results concerning the transition into teletherapy are available as preprint. (Huscsava et al., 2020) Thoughts expressed by psychiatrists and psychologists obtained in informal discussions are integrated where appropriate to provide a comprehensive overview.

Structured qualitative data analysis was conducted following the principles of Grounded Theory (11) using the computerised qualitative data analysis tool ATLAS.ti 8 (ATLAS.ti Scientific Software Development GmbH, Berlin, Germany).

\section{Results}

\subsection{Patients' topics}

To get an idea on what moved our patients when lockdown measures in Vienna were at their maximum, including homeschooling, part-time work, and restrictions on physical contact, Figure 1 provides a word cloud drawn from asking the patients to freely express current thoughts. To obtain the presented word cloud, patients' answers were reduced to nouns, verbs (excluding auxiliary verbs), adverbs and adjectives. Translations were carried out by a study member, checked with Merriam-Webster Thesaurus and cross-checked with Collins Dictionary. (Collins Wörterbuch, 2020; Merriam-Webster Thesaurus, 2020) The builtin word-cloud-function of ATLAS.ti 8 was used to obtain the cloud-presentation of the initial word list.A higher word count is indicated by a larger font. As can be seen, thoughts about the future and family, followed by those relating to friends and school preoccupied our patients the most. Teletherapy was provided by staff well-trained in the provision of non-teletherapy treatments, who have not been asked to give their thoughts in the same manner. It can be assumed though, that the picture might coincide in some points. Numerous investigations, dating from prior events (e.g. disasters, epidemics and pandemics), and also stemming from the current COVID-19 pandemic, assessed mental health consequences for health care workers, focusing on those who were "frontline workers". (Hu \& Chen, 2020; Lu et al., 2006; Lung et al., 2009) Mental health professionals providing teletherapy during the current pandemic suffered as many restrictions as other medical staff and the rest of the population, but have not been specifically investigated as a subgroup. This has to be taken into account when discussing teletherapy in this situation. Working with a novel technology in an environment of - global insecurity, and situationally reduced exchange with colleagues, is rather challenging. In this light, it seems even more surprising (or convincing) that the patients we interviewed rated teletherapy to be on average between rather good and mediocre, as compared to the face-to-face contacts they were used to (3.2 on a 5 -point-Likert scale, 1 indicating "bad" and 5 indicating "good").

\subsection{Main arguments in favor of teletherapy}

Most patients expressed great relief about the fact that they still had access to continuous treatment while basically everything else was locked down. In fact, to date not a single patient we followed up dropped out of treatment during the pandemic. This is not straightforward, nor is the seamless continuation of therapy. Given our observations, the patients' feedback, and opinions and experiences expressed by colleagues in informal discussions, the content of many therapeutic processes changed in the sense that it became somewhat more superficial and oriented toward every-day life. However, in all cases, it was possible to keep in touch. This was the case regardless of the device used, a surprising result, because it was assumed that seeing one's therapist on a computer screen would be different than seeing them on a 5-inch cell phone. Many patients also mentioned that teletherapy was an anchor in their week, something they could be sure of while everything else felt "endless" and "surreal". Furthermore, patients commented that it was easier to fit teletherapy into their schedule, because they did not have to spend time travelling to the treatment centre, and some also found it to be more flexible. While a number of patients mentioned that although they would prefer face-to-face contact, teletherapy gives them the opportunity to "really see" their therapists, because that way no one had to wear a mask. Most patients also appreciated the chat function of the video-call system as an extra facility to note thoughts and questions and to keep track of appointments. 


\subsection{Main arguments against teletherapy}

The arguments against teletherapy varied between the patients who rated teletherapy as rather bad or bad as compared to those who rated it at least as mediocre. Patients who rated teletherapy more highly focused on technical aspects. It should be noted that all patients and therapists complained about technical issues, most notably problems concerning the internet connection. Other negative aspects mentioned by most patients centered around the feeling of a lack of privacy, even if they had their own room or private space. They also highlighted that they felt they could not be quite as open. Some patients indicated that they experienced a high level of distraction, e.g. due to alerts on any of their devices, such as their cell phone, notebook, or tablet, or because of people entering the room. Those who rated teletherapy as rather bad or bad stressed that they felt unsafe and were more bothered by not having a direct face-to-face counterpart.

\section{Conclusion and discussion}

The reported results are drawn from a small sample, as well as informal discussions with colleagues, and are thus hardly generalizable. Nevertheless, they provide some important insights worth highlighting. Prior studies on teletherapy could show that results of assessments match those from direct contacts (Diamond \& Bloch, 2010) and overall acceptance and satisfaction by patients is good (Pesämaa et al., 2004). Also, barriers to use teletherapy have been evaluated, where important aspects seem to lie on the providers' side (e.g. policy, reimbursements). (Cowan et al., 2019) Although diverse facets of teletherapy have been explored, most studies focused on service provision in remote and rural areas and on teletherapy as an explicit offer and choice. Also, the transition of well-established face-to-face treatment into teletherapy with the same therapist has not been addressed explicitly. Our study focused on these aspects and could show that teletherapy generally seems to be a feasible option for adolescent psychiatric outpatients, even if they are in intensive treatment, although not all may benefit equally from it. For a continuation of teletherapeutic offers, infrastructure definitely needs to be adapted (high speed up- and download internet connections and the guarantee of unlimited data transfer). Interestingly, the size of the display was not a relevant factor for our patients, which indicates that teletherapy could potentially increase access to services in remote areas, because of the availability of mobile phones. Concerning the aspect of distraction, it might be sensible to set up a "teletherapy contract" with patients, guardians, or families, which includes commitments to turn off alerts during therapy and to not be disrupted by family members. Some therapists mentioned they felt more insecure in having limited options to act in the event of crises and escalations, especially in case of an unstable internet connection. It might be useful to discuss this aspect with patients and guardians beforehand and sharpen the guardians' awareness, have their telephone numbers at hand, and discuss a solid and up-to-date crisis plan with both parties. Extra communication paths like

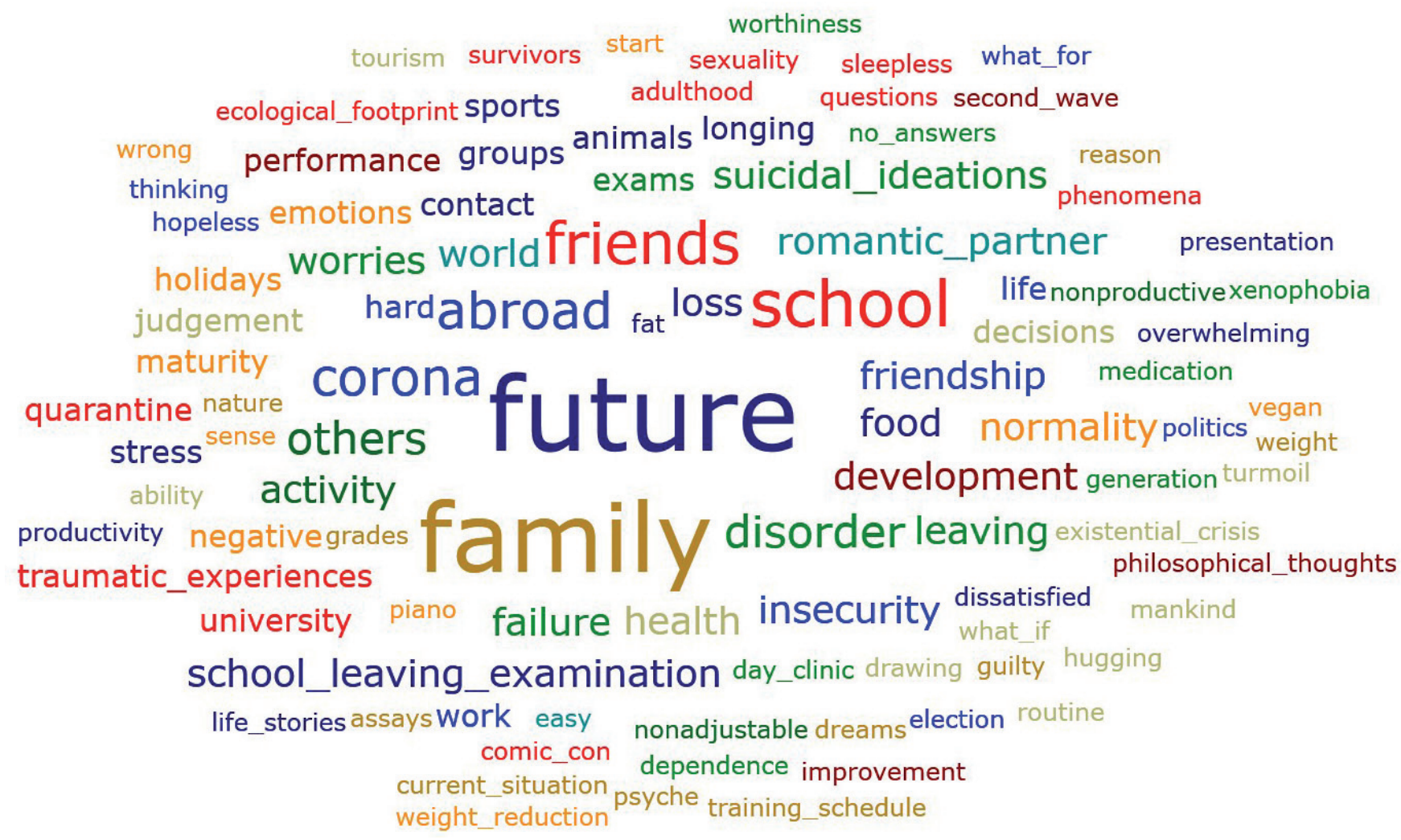

Figure 1. Word cloud indicating main themes preoccupying patients during lockdown 
chats were also welcomed and used frequently by our patients. The possible flip side of potential overuse of the patients did not prove true in our observations, so that these pathways can also be seen as a chance, e.g. for interim boosters. Although teletherapy with children and adolescents is still in its infancy, the COVID-19 pandemic led to a massive expansion of the application of these technologies, resulting in an increased and growing body of experience. Further investigations are necessary to develop guidelines for a proper framework for teletherapy and on how to distinguish patients who will benefit from teletherapy from those who should be offered face-to-face contact. Furthermore, teletherapy should be included in the training curricula for psychiatrists, psychologists and psychotherapists.

\section{References}

Collins Wörterbuch. (2020). HarperCollins Publishers. https://www. collinsdictionary.com/de/worterbuch/englisch-deutsch

Corbin, J. M., \& Strauss, A. L. (2008). Basics of Qualitative Research. Techniques and Procedures for Developing Grounded Theory (3rd ed.). Sage.

Cowan, K. E., McKean, A. J., Gentry, M. T., \& Hilty, D. M. (2019, 2019/12/01/). Barriers to Use of Telepsychiatry: Clinicians as Gatekeepers. Mayo Clinic Proceedings, 94(12), 2510-2523. https://doi.org/ https://doi.org/10.1016/j.mayocp.2019.04.018

Cui, Y., Li, Y., Zheng, Y., Chinese Society of, C., \& Adolescent, P. (2020, May 11). Mental health services for children in China during the COVID-19 pandemic: results of an expert-based national survey among child and adolescent psychiatric hospitals. Eur Child Adolesc Psychiatry. https://doi.org/10.1007/s00787-020-01548-x

Diamond, J. M., \& Bloch, R. M. (2010). Telepsychiatry Assessments of Child or Adolescent Behavior Disorders: A Review of Evidence and Issues. Telemedicine and e-Health, 16(6), 712-716. https://doi. org/10.1089/tmj.2010.0007

Druss, B. G. (2020, Apr 3). Addressing the COVID-19 Pandemic in Populations With Serious Mental Illness. JAMA Psychiatry. https:// doi.org/10.1001/jamapsychiatry.2020.0894

Fegert, J. M., Vitiello, B., Plener, P. L., \& Clemens, V. (2020). Challenges and burden of the Coronavirus 2019 (COVID-19) pandemic for child and adolescent mental health: a narrative review to highlight clinical and research needs in the acute phase and the long return to normality. Child Adolesc Psychiatry Ment Health, 14, 20. https://doi. org/10.1186/s13034-020-00329-3

Hu, Z., \& Chen, B. (2020, 2020-June-05). The Status of Psychological Issues Among Frontline Health Workers Confronting the Coronavirus Disease 2019 Pandemic [Opinion]. Frontiers in Public Health, 8(265). https://doi.org/10.3389/fpubh.2020.00265

Huscsava, M. M., Scharinger, C., Plener, P. L., \& Kothgassner, O. D. (2020). "Bridge over troubled water": A first look at adolescent psychiatric outpatients transited from regular care to teletherapy during the COVID-19 outbreak using quantitative and qualitative analyses. PREPRINT. https://doi.org/10.21203/rs.3.rs-40679/v1

Kavoor, A. R. (2020, Apr 8). COVID-19 in People with Mental Illness: Challenges and Vulnerabilities. Asian J Psychiatr, 51, 102051. https:// doi.org/10.1016/j.ajp.2020.102051

Liu, S., Yang, L., Zhang, C., Xiang, Y.-T., Liu, Z., Hu, S., \& Zhang, B. (2020). Online mental health services in China during the COV-
ID-19 outbreak. The Lancet Psychiatry, 7(4), e17-e18. https://doi. org/10.1016/s2215-0366(20)30077-8

Lu, Y. C., Shu, B. C., Chang, Y. Y., \& Lung, F. W. (2006). The Mental Health of Hospital Workers Dealing with Severe Acute Respiratory Syndrome. Psychotherapy and Psychosomatics, 75(6), 370-375. https://doi.org/10.1159/000095443

Lung, F. W., Lu, Y. C., Chang, Y. Y., \& Shu, B. C. (2009, Jun). Mental Symptoms in Different Health Professionals During the SARS Attack: A Follow-up Study. Psychiatr Q, 80(2), 107-116. https://doi. org/10.1007/s11126-009-9095-5

Merriam-Webster Thesaurus. (2020). Merriam-Webster Inc. https:// www.merriam-webster.com/

Pesämaa, L., Ebeling, H., Kuusimäki, M.-L., Winblad, I., Isohanni, M., \& Moilanen, I. (2004). Videoconferencing in child and adolescent telepsychiatry: a systematic review of the literature. Journal of Telemedicine and Telecare, 10(4), 187-192. https://doi.org/ $10.1258 / 1357633041424458$

Torous, J., Jan Myrick, K., Rauseo-Ricupero, N., \& Firth, J. (2020, Mar 26). Digital Mental Health and COVID-19: Using Technology Today to Accelerate the Curve on Access and Quality Tomorrow. JMIR Ment Health, 7(3), e18848. https://doi.org/10.2196/18848

Torous, J., \& Wykes, T. (2020). Opportunities From the Coronavirus Disease 2019 Pandemic for Transforming Psychiatric Care With Telehealth. JAMA Psychiatry. https://doi.org/10.1001/jamapsychiatry.2020.1640

Wind, T. R., Rijkeboer, M., Andersson, G., \& Riper, H. (2020, Apr). The COVID-19 pandemic: The 'black swan' for mental health care and a turning point for e-health. Internet Interv, 20, 100317. https://doi. org/10.1016/j.invent.2020.100317

Yao, H., Chen, J.-H., \& Xu, Y.-F. (2020). Patients with mental health disorders in the COVID-19 epidemic. The Lancet Psychiatry, 7(4), e21. https://doi.org/10.1016/S2215-0366(20)30090-0

Zho, X., Snoswell, C. L., Harding, L. E., Bambling, M., Edirippulige, S., Bai, X., \& Smith, a. A. C. (2020). The Role of Telehealth in Reducing the Mental Health Burden from COVID-19. Telemedicine and eHealth, 26(4), 377-379. https://doi.org/10.1089/tmj.2020.0068

\section{${ }^{*}$ Corresponding Author}

\section{Mercedes M. Huscsava, MD}

Department of Child and Adolescent Psychiatry

Medical University of Vienna

Währinger Gürtel 18-20, 1090 Wien

T: +43 (0)1 40400-30140, E: mercedes.huscsava@meduniwien.ac.at

\section{Declarations}

\section{Conflict of interest}

None of the authors has competing interests in connection with this study.

None of the authors holds stocks of pharmaceutical companies.

\section{Funding}

This research has been conducted with financial support from the Medical Scientific Fund of the Mayor of the City of Vienna (\#COVID066). 